\title{
Effects of transcranial direct current stimulation on the working memory of post-stroke people: an integrative review
}

\author{
Edson Meneses da Silva Filho1, Jéssica Andrade de Albuquerque², Karime Andrade Mescouto', \\ Rodrigo Pegado de Abreu Freitas ${ }^{3}$
}

\begin{abstract}
Background: Stroke is a chronic disease that is becoming more common in all the world causing damage to people affected, for instance, cognitive deficits. The Transcranial Direct Current Stimulation (tDCS) is a form of treatment that modulates specific areas of the nervous system. Objective: The objective of this integrative review was to analyze the effectiveness of Transcranial Direct Current Stimulation (tDCS) on the working memory (WM) in post-stroke patients. Method: This review included articles that analyzed the effects of tDCS on human beings with a diagnosis of stroke and used as outcomes: verbal, visual, spatial or auditory WM. The assessment of studies elegibility was performed by two independent researchers from 8 databases: Cochrane Library via Wiley - CENTRAL, PubMed, LILACS, SCIELO, Web of Science, Scopus, CINAHL and PEDro following PRISMA guideline. Results: Three articles were included in the qualitative synthesis presenting a total of 66 participants ( 32 male and 34 female), 36 ischemic stroke and 30 hemorrhagic stroke with an average time of 42 days. Interventions were done on the areas F3/F4 (dorsolateral part of the prefrontal cortex) and T3/T4 (temporal anterior lobe) with $2 \mathrm{~mA}$ current intensity for 30 minutes duration. The outcomes analyzed: verbal, visual, spatial, and auditory WM showed significant improvements after the use of tDCS. Conclusion: The limitations of this review were study designs, number of participants, lack of standardized interventions and short period of follow-up. tDCS showed satisfactory results on the WM of post-stroke individuals, but it is needed to be cautious due to the methodological quality of the articles. It is registered in the Prospective Register of Systematic Reviews - PROSPERO with registration number CDR42016048050.
\end{abstract}

Keywords: Stroke; Transcranial Direct Current Stimulation; Memory

\section{INTRODUCTION}

Stroke is a disease that has a great impact worldwide specially in developing countries ${ }^{(1)}$. Factors such as age, Alzheimer's, female gender and hypertension increase the risk for developing stroke ${ }^{(2)}$. In addition, silent infartcs have been associated with increased risk of symptomatic stroke and cognitive impairment ${ }^{(3)}$.

One of the locations associated with cognitive deficits in stroke is located in the Working Memory (WM). This type of memory is used for temporary storage of information, being responsible for various processes, such as comprehension, language, learning and consolidation of long-term memory ${ }^{(4)}$ and according to the classical model of Baddeley and Hitch ${ }^{(5)}$ it is composed of two other temporary memory, verbal and visuospatial, which are often affected after stroke.

Transcranial Direct Current Stimulation (tDCS) is a nonivasive and safe technique used to improve cognitive impairments, which acts by modulating specific areas of the central nervous system that modifies neuronal electrical membrane potentials and its activity ${ }^{(6)}$. This technique has also been directed to the improvement of the WM functions in different populations, specifically stimulating the dorsolateral area of the prefrontal cortex of subjects ${ }^{(7-11)}$. Although, there is a growing number of studies that use tDCS to improve patients cognitive functions, there are still insufficient searches in specific populations, such as post-stroke patients with WM impairment.

The knowledge about the proper use of tDCS in post-stroke people is important for professionals to manage this technique in a standardized manner, giving individuals the maximum benefits of treatment. It is hypothesized that tDCS shows satisfactory results on the WM in post-stroke individuals.

The question on which this review was based for the construction of the PICO was: Does the transcranial direct current stimulation interferes on the working memory of post-stroke adults? P - adults, stroke; I - transcranial direct

Corresponding author: Edson Meneses da Silva Filho. Rua Andrade Bezerra, 78, Centro - Timbaúba - PE, Brazil. CEP: $55870-000$. Phone: 005581994706661. Email: meneses.edson@yahoo.com.br

${ }^{1}$ Physiotherapist, master student in Science of Rehabilitation - Universidade Federal do Rio Grande do Norte - UFRN, Faculty of Health Sciences at Trairi, Santa Cruz, Rio Grande do Norte, Brazil.

Full list of author information is available at the end of the article.

Financial source: There is not.

Submission date 06 June 2017; Acceptance date 22 November 2017; Publication date 30 November 2017 
current stimulation; C - transcranial direct current stimulation (sham) or active control (cognitive training); O - working memory. The verbal WM was adopted as primary outcome, the visual, spatial and auditory WM were considered as secundary outcomes.

Thus, the purpose of this integrative review was to analyse the effects of transcranial direct current stimulation on the working memory of post-stroke individuals compared to any active or passive control.

\section{MATERIALS AND METHODS}

\section{Protocol and registration}

This review followed the recommendations of the Preferred Reporting Itens for Systematic Reviews and Meta-Analyses (PRISMA) ${ }^{(12)}$ and it is registered in the Prospective Register of Systematic Reviews - PROSPERO with registration number CDR42016048050.

\section{Eligibility criteria}

This review included articles that showed the following criteria: studies with patients $>18$ years of age with a diagnosis of stroke, without gender restriction, to be written in any language and year of publication showing the effects of transcranial direct current stimulation (tDCS) in post-stroke individuals and to have used as outcome verbal, visual spatial and auditory Working Memory (WM). Active tDCS (unilateral/bilateral, anodic/cathodic) or active tDCS associated with any cognitive training that influenced on the WM was compared to sham tDCS intervention or any active or passive control. It was considered as active tDCS, long term application (greater than 1 minute) on the injured hemisphere or bilateral and defined as sham tDCS, positioning the electrodes without current application or stimulus of short duration (less than 1 minute). This is considered a suficient time for the current sensation on the scalp disappears ${ }^{(13)}$.

\section{Search strategy}

The search strategies were made according to the specificities (language, synonyms, truncation and descriptors) of the databases used to search the articles. The databases used for collection of the articles were the Cochrane Library via Wiley - CENTRAL, PubMed, LILACS, SCIELO, Web of Science, Scopus, CINAHL and PEDro. To identify more published, unpublished and ongoing studies a search was done in clinical trials registers, reference lists of included studies and was made contact with the authors, if needed. The last search was conducted on october 18, 2016.

\section{Data colletction and analysis}

Initially, a simple search was done by two independent researchers in the databases mentioned with the insertion of descriptors and Boolean operators according to the official language and accepted nomenclature (mesh). Then, a sensitized search was done through the inclusion of descriptor's synonyms and its categories.

\section{Studies selection}

The titles, abstracts and pottentialy relevant full texts were screened by the researchers. Articles that met inclusion criteria were stored and during the consensus meeting the researchers discussed which study would take part of this integrative review. A third investigator was contacted in case of desagreements.

\section{Data collection process}

Data were independently extracted from the included studies by both researchers. A formulary was created in order to obtain the largest possible number of data as: identification and study design, data randomization, sample (country, age and gender), stage of disease, intervention groups, allocation concealment, follow-up, bliding, statistical power, inclusion and exclusion criteria, outcomes, selective description and summary measures. The comparison among the data obtained was done in the consensus meeting. Authors were only contacted to alucidate any unclear information about the article.

\section{Risk of bias assessment}

The tool risk of bias table made by the Cochrane Collaboration was used for the analysis of risk of bias of the studies ${ }^{(14)}$. This analysis was done by two researchers independently and in the consensus meeting was discussed, evaluated and decided the risk of bias from each study. The third investigator was contacted, if there was some desagreement.

\section{RESULTS}

\section{Articles Selection}

A total of 3.549 articles were initially identified. After reading the titles/abstracts, 3.497 articles were excluded because they did not met the inclusion criteria, remaining a total of 52 elegible articles. Ultimately, only 3 articles were suitable for quality assessment after reading the full texts, as represented in figure 1.

\section{Characteristics of the articles}

The description of all the studies, objectives, sample characteristics, interventions, outcomes and main results are in Table 1.

A total of 66 participants (32 male and 34 female), 36 ischemic stroke and 30 hemorrhagic stroke with an average time of 42 days $^{(15-17)}$. The interventions were done on the areas F3/F4 (dorsolateral part of the prefrontal cortex) and T3/T4 (temporal anterior lobe) for stimulation in accordance with the international $10-20$ system $\mathrm{EEG}^{(18)}$ with $2 \mathrm{~mA}$ current intensity 
for 30 minutes duration. The follow-up was pre/post treatment in all studies, but they differ in the session number. Yun et al. ${ }^{(15)}$ presented a total of 15 sessions being $3 x /$ week, Jung et al. ${ }^{(16)}$ did only 2 sessions with a 48 hours interval and Park et al. ${ }^{(17)}$ didn't report the amount of sessions.

\section{Risk of bias assessment}

Park et al. ${ }^{(17)}$ did not describe properly the randomization, allocation concealment, blidind: searcher, participants and outcomes, however, the data were complete, there were not selective description and other bias. Jung et al. ${ }^{(16)}$ did not randomize the sample. The allocation concealment, blidind searcher and participants were not described properly, besides bliding outcomes was not done. There was incomplete outcome data and other bias, but selective description did not occur. Yun et al. ${ }^{(15)}$ did not describe properly the randomization, allocation concealment, blidind: searcher, participants and outcomes, however, the data was complete, there was not selective description and other bias. The risk of bias of the articles is specified in Figure 2.

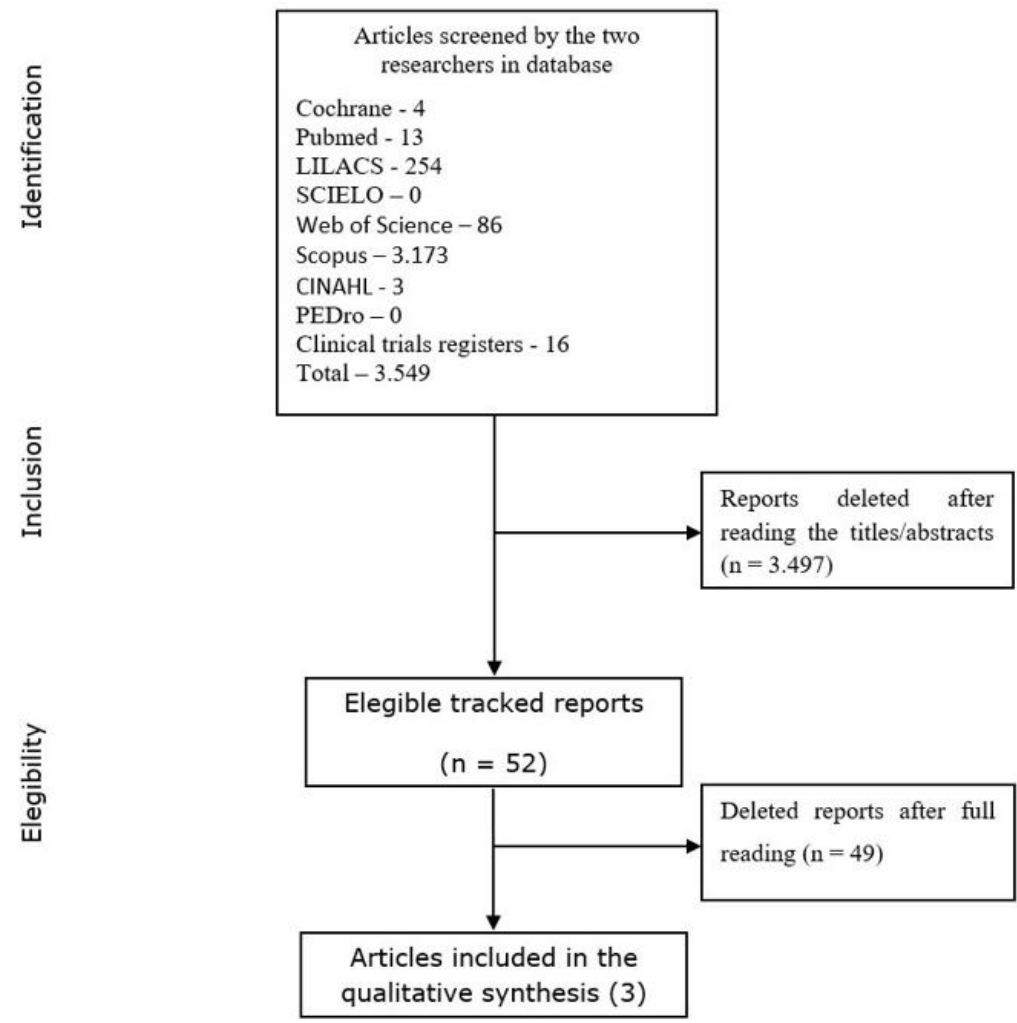

Figure 1. Flowchart of selected articles

\section{Risk of bias table}

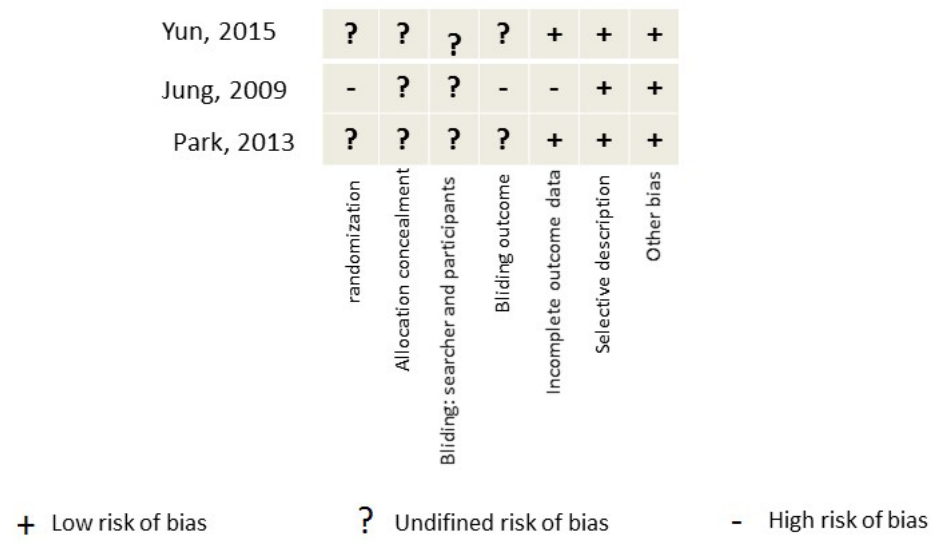

Figure 2. Bias risk analysis of the articles included in the review. 


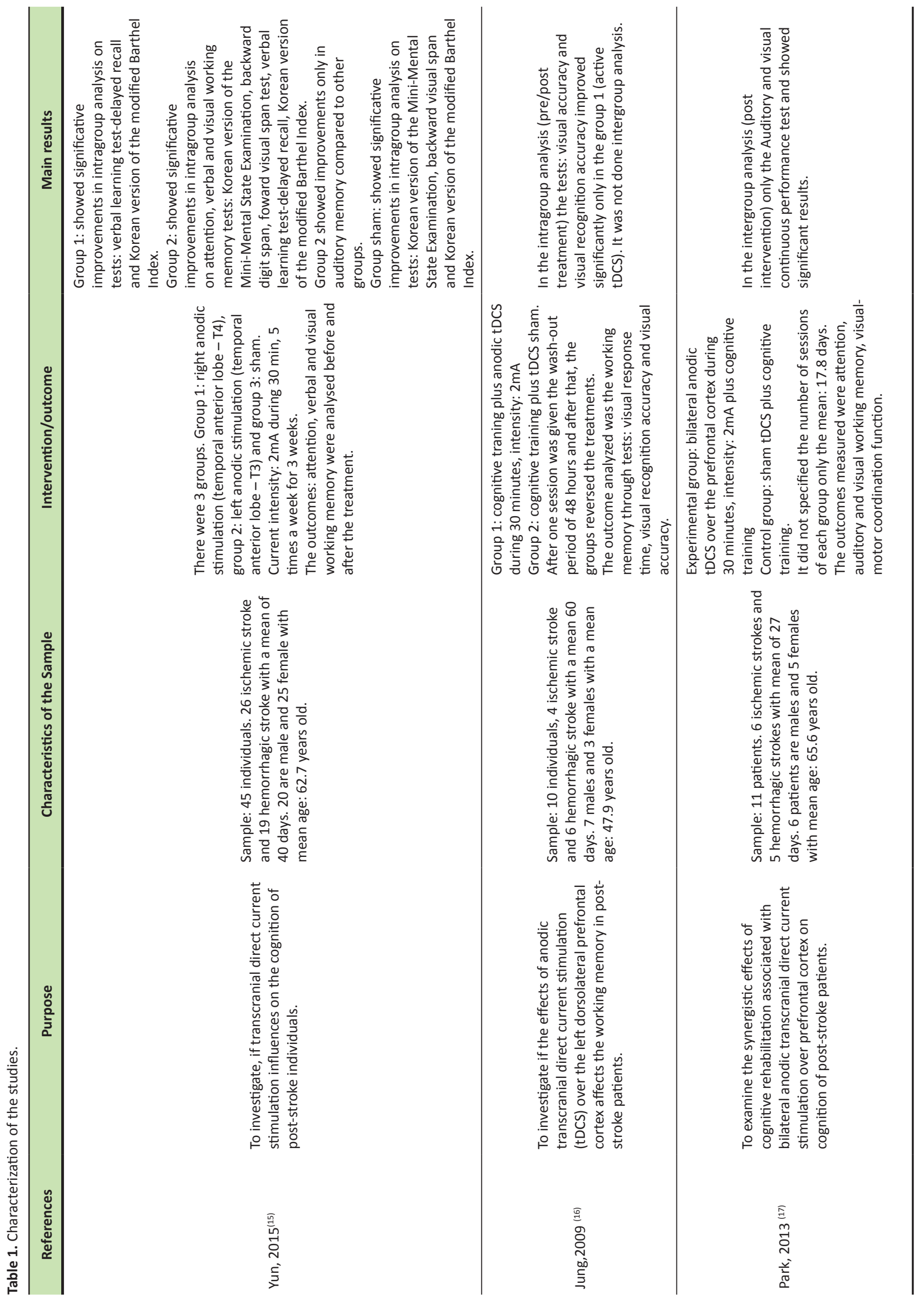




\section{DISCUSSION}

This is the first integrative review of studies that analysed Transcranial Direct Current Stimulation (tDCS) in post-stroke individuals using as outcome the Working Memory (WM). Moreover, it is also important to mention that there are few studies that have used this type of intervention in post-stroke patients, thus justifying the small number of articles present in this review.

The three studies that adopted this approach showed undefined risk of bias, according to the methodological evaluation of this review. They showed different interventions, which differed in the number of sessions and study design, thus making difficult compare them. Other variables, such as intensity of current, stimulated area, stimulation time and methods used to measure the outcomes were similar, corroborating with protocols used by other authors in different populations that investigated the same outcomes ${ }^{(19,20)}$.

It is known that after stroke may occur a change in synaptic homeostasis, which affects individuals' cognition ${ }^{(21)}$. In the present study the verbal and visual WM showed significant improvements after the use of tDCS, supporting the results found in diverse population by other authors ${ }^{(22,23)}$. One possible explanation is that the modulation of membrane action potencial induced by tDCS could limit this homeostasis lost, therefore mantaining the cognitive functions ${ }^{(24)}$. A brief episode of strong synaptic activation after stimulation has demonstrated improvements in neural transmission and neuroplasticity, hence improving memory and learning ${ }^{(25)}$.

The spatial WM showed significant results after the use of tDCS possibly because of neurons activation patterns enhancement, through the late time window that generated improvements in memory functionality ${ }^{(26)}$. Another reason is based on the fact that some authors have found evidence of a separation of a WM information division of object and space in parietal and temporal lobe, while the dorsolateral part of the prefrontal cortex (DLPFC) was important for both types of information ${ }^{(27)}$. In a study about temporal dynamics and interactions within many different areas it was found interferences after stimulation between the DLPFC and parietal areas on tasks involving spatial $\mathrm{WM}^{(28)}$, so it is assumed that tDCS may have influenced the neural network associated with spatial WM both locally and in distant places ${ }^{(29)}$.

Some authors have suggested that the DLCPF plays an important role in the planning and proper execution of motor response during WM based tasks ${ }^{(30,31)}$, in addition, the declarative memory $^{(32)}$, emotional(33), and attention ${ }^{(34)}$ are also being used for cognitive rehabilitation with the tDCS. After stimulation of DLPFC area, significant results were found, through analysis of visual and spatial WM outcomes, being in accordance with previous studies on other populations ${ }^{(35-37)}$. Other areas such as posterior ${ }^{(38)}$ and lateral parietal ${ }^{(39)}$ are also being used as sities of stimulation, in an attempt to assist in cognitive performance after injury.
Some authors were able to observe improvements in auditory WM after stimulation ${ }^{(40,41)}$. Following a brain injury, individuals often have deficits in specific anatomical listening areas including Wernicke's and surrounding areas ${ }^{(42,43)}$. tDCS provides changes in cortical excitability and an increase of cognitive performance is observed when followed by specific training ${ }^{(44)}$, therefore justifying the improvements found in WM auditory outcome. The result in the auditory continuous performance test presented in this review should be viewed with caution given that the pre-treatment groups were not homogeneous.

Among the limitations encountered by this review we can highlight the studies designs that made impossible conduct a meta-analysis. Also the number of participants, lack of standardized intervention and a small follow-up.

\section{CONCLUSIONS}

The use of transcranial direct current stimulaton in post-stroke individuals demonstrated improvements on the working memory after treatment, but better design studies that have a longer follow-up, standardized intervention and a large sample are needed.

\section{AUTHORS' CONTRIBUTION}

EMSF, JAA and KAM participated in the idealization of the study, data collection, analysis/interpretation and article writing; EMSF and JAA participated in data collection; RPAF participated, in the condition of guiding, the idealization of the study, analysis, interpretation of data and article's writing.

\section{CONFLICTS OF INTEREST}

There is not

\section{AUTHOR DETAILS}

2 Psychologist, PhD student in Social Psycholigist - Universidade Federal da Paraíba - UFPB, João Pessoa, Brazil.

${ }^{3}$ Physiotherapist (PhD), Universidade Federal do Rio Grande do Norte, Faculty of Health Sciences at Trairi, Santa Cruz, Rio Grande do Norte, Brazil.

\section{REFERENCES}

1. Truelsen T, Heuschmann PU, Bonita, R. Standard method for developing stroke registers in low-income and middle income countries: experiences from a feasibility study of a stepwise approach to stroke surveillance (STEPS Stroke). The Lancet Neurology. 2007;6:134-139.

2. Shehadri S, Beiser A, Kelly-Hayes M, Kase CS, Au R, Kannel WB, Wolf PA. The lifetime risk of stroke: estimates from the Framingham Study. Stroke. 2006;37:345-350.

3. Vermeer SE, Prins ND, Den Heijer T, Hofman A, Koudstaal PJ, Breteler MM. Silent brain infarcts and the risk of dementia and cognitive decline. N Engl J Med. 2003;348:1215-1222.

4. Baddeley A. Working memory, thought, and action. In: D'Esposito M, Schacter D, Driver J, et al., editors. Oxford: Oxford University Press, 2007.

5. Baddeley A, Hitch GJ. Working memory. In: Bower GA. Recent Advances in Learning and Motivation. New York, Academic Press. 1974;8:47-90.

6. Nitsche MA, Liebetanz D, Antal A, Lang N, Tergau F, Paulus W: Modulation of cortical excitability by weak direct current stimulation technical, safety and functional aspects. Suppl Clin Neurophysiol. 2003;56:255-276. 
7. Coffman BA, Clark VP, Parasuraman R. Battery powered thought: Enhancement of attention, learning, and memory in healthy adults using transcranial direct current stimulation. Neurolmage. 2014;85:895-908.

8. Makovski T, Lavidor M. Stimulating occipital córtex enhances visual working memory consolidation. Behavioural Brain Research. 2014; 275:84-87.

9. Meiron O, Lavidor M. Unilateral Prefrontal Direct Current Stimulation Effects are Modulated by Working Memory Load and Gender. Brain Stimulation. 2013; 6:440-447.

10. Berryhill ME, Jones KT. tDCS selectively improves working memory in older adults with more education. Neuroscience Letters. 2012;521:148-151.

11. Jo JM, Kim Y, Ko M, Ohn SH, Joen B, Lee KH. Enhancing the Working Memory of Stroke Patients Using tDCS. Am. J. Phys. Med. Rehabil. 2009;88:5

12. Moher D, Liberati A, Tetzlaff J, Altman DG. Preferred reporting items for systematic reviews and meta-analyses: the PRISMA statement. Ann Intern Med. 2009;151(4):264-9.

13. Gandiga PC, Hummel FC, Cohen LG. Transcranial DC stimulation (tDCS): a tool for double-blind sham-controlled clinical studies in brain stimulation. Clinical Neurophysiology. 2006;117:845-50.

14. Higgins JPT, Altman DG. Assessing risk of bias in included studies. In: Higgins JPT, Green S, eds. Cochrane handbook for systematic reviews of interventions. Wiley. 2008:187-241.

15. Yun GJ, Chun MH, Kim BR.The Effects of Transcranial Direct-Current Stimulation on Cognition in Stroke Patients. Journal of Stroke. 2015;17(3):354-358.

16. Jung JM, Kim YH, Ko MH, Ohn SH, Joen B, Lee KH. Enhancing the working memory of stroke patients using tDCS. Am Journal Phys Med Rehabil. 2009;88:404-409.

17. Park SH, Koh EJ, Choi HY, Ko MH. A Double-Blind, Sham-Controlled, Pilot Study to Assess the Effects of the Concomitant Use of Transcranial Direct Current Stimulation with the Computer Assisted Cognitive Rehabilitation to the Prefrontal Cortex on Cognitive Functions in Patients with Stroke. Journal of Korean Neurosurg Soc. 2013;54:484-488.

18. Da Silva AF, Volz MS, Bikson M, Fregni F. Electrode Positioning and Montage in Transcranial Direct Current Stimulation. J. Vis. Exp. 2011;(51):2744.

19. Ohn SH, Park C, Yoo W, Ko M, Choi KP, Kim G. Time-dependent effect of transcranial direct current stimulation on the enhancement of working memory. Neuroreport. 2008;19(1):43-7.

20. Mylius V, Jung M, Menzler K, Haag A, Khader PH, Oertel WH, Rosenow F, Lefaucheur JP. Effects of transcranial direct current stimulation on pain perception and working memory. Eur J Pain. 2012;16:974-982.

21. Thickbroom G, Mastaglia F. Plasticity in neurological disorders and challenges for non-invasive brain stimulation. Journal of NeuroEngineering and Rehabilitation. 2009;6:4.

22. Park SH, Seo JH, Kim YH, Ko MH. Long-term effects of transcranial direct current stimulation combined with computer-assisted cognitive training in healthy older adults. Neuroreport. 2014;25(2):122-6.

23. Hussey EK, Ward N, Christianson K, Kramer AF. Language and Memory Improvements following tDCS of Left Lateral Prefrontal Cortex. PLoS ONE. 2015;10(11).

24. Rodger J, Mo C, Wilks T, Dunlop SA, Sherrard RM. Transcranial pulsed magnetic field stimulation facilitates reorganization of abnormal neural circuits and corrects behavioral deficits without disrupting normal connectivity. FASEB. 2012;26:1596-1606.

25. Floel A, Cohen LG. Contribution of noninvasive cortical stimulation to the study of memory functions. Brain Res Rev. 2007;53:250-259.

26. Lu Y, Wang C, Chen C, Xue G .Spatiotemporal Neural Pattern Similarity Supports Episodic Memory. Current Biology. 2015;25:780-785.
27. Oliveri M, Turriziani P, Carlesimo GA, Koch G, Tomaiuolo F, Panella M.. Parieto-frontal interactions in visual-object and visual-spatial working memory: evidence from transcranial magnetic stimulation. Cereb. Cortex. 2001;11(7):606-618.

28. Koch G, Oliveri M, Torriero S, Carlesimo GA, Turriziani P,Caltagirone C. rTMS evidence of different delay and decision processes in a fronto-parietal neuronal network activated during spatial working memory. Neurolmage. 2005;24(1):34-39.

29. Lang N, Siebner HR, Ward NS, Lee L, Nitsche MA, Paulus W. How does transcranial DC stimulation of the primary motor cortex alter regional neuronal activity in the human brain? Eur J Neurosci. 2005;22:495-504.

30. Zaehle T, Sandmann P, Thorne JD, Jäncke L, Herrmann CS. Transcranial direct current stimulation of the prefrontal cortex modulates working memory performance: combined behavioural and electrophysiological evidence. BMC Neuroscience. 2011;12:2.

31. Curtis CE, Rao VY, D’Esposito M. Maintenance of spatial and motor codes during oculomotor delayed response tasks. J Neurosci. 2004;24:39443952.

32. Pisoni A, Turi Z, Raithel A, Ambrus GG, Alekseichuk I, Schacht A, Paulus W, Antal A. Separating Recognition Processes of Declarative Memory via Anodal tDCS: Boosting Old Item Recognition by Temporal and New Item Detection by Parietal Stimulation. PLoS One. 2015;10(3).

33. Morgan HM, Davis NJ, Bracewell RM . Does Transcranial Direct Current Stimulation to Prefrontal Cortex Affect Mood and Emotional Memory Retrieval in Healthy Individuals? PLOS ONE. 2014;9:3.

34. Reinhart RMG, Woodman GF. Enhancing long-term memory with stimulation tunes visual attention in one Trial. PNAS. 2015;112(2):625630.

35. Richmond L, Wolk D, Chein JM, Olson IR. Transcranial Direct Current Stimulation Enhances Verbal Working Memory Training Performance over Time and Near Transfer Outcomes .Journal of Cognitive Neuroscience. 2014;26(11):2443-2454.

36. Jones KT, Stephens JA, Alam M, Bikson M, Berryhill ME. Longitudinal Neurostimulation in Older Adults Improves Working Memory. PLoS ONE. 2015;10(4).

37. Andrews SC, Hoy KE, Enticott PG, Daskalakis ZJ, Fitzgerald PB .Improving working memory: the effect of combining cognitive activity and anodal transcranial direct current stimulation to the left dorsolateral prefrontal cortex. Brain Stimulation.2011;4:84-9.

38. Kevin T, Filiz G, Marian E. Berryhill Enhanced long-term memory encoding after parietal. Neurostimulation Exp Brain Res. 2014;232(12):4043-4054.

39. Jane X. Wang L, Joel L. Voss Long-lasting enhancements of memory and hippocampal-cortical functional connectivity following multiple-day targeted noninvasive stimulation. Hippocampus. 2015;25(8):877-883.

40. Fertonani A, Pirulli C, Miniussi C. Random Noise Stimulation Improves Neuroplasticity in Perceptual Learning. The Journal of Neuroscience. 2011;31(43):15416-15423.

41. Ladeira A, Fregni F, Campanha C, Valasek CA, Ridder D, Brunoni AR, Boggio PS. Polarity-Dependent Transcranial Direct Current Stimulation Effects on Central Auditory Processing. PLoS ONE 2011; 6:9.

42. Wittmann M, Burtscher A, Fries W, Steinbüchel N. Effects of brainlesion size and location on temporal order judgement. Neuroreport. 2004;15(15):2401-5.

43. Lewandowska M, Piatkowska-Janko E, Bogorodzki P, Wolak T, Szelag E. Changes in $\mathrm{FMRI} B O L D$ response to increasing and decreasing task difficulty during auditory perception of temporal order. Neurobiol Learn Mem. 2010;94(3):382-91.

44. Javadi AH, Cheng P. Transcranial Direct Current Stimulation (tDCS) Enhances Reconsolidation of Long- Term Memory. Brain Stimulation.2012;1-7. 